\title{
Riesgo de adicción a redes sociales e Internet y su relación con habilidades para la vida y socioemocionales en una muestra de estudiantes de bachillerato del municipio de Envigado
}

\author{
Risk of addiction to social networks and the Internet and its \\ relationship with life and socio-emotional skills in a sample \\ of high school students from the municipality of Envigado
}

\section{(U) PSICOGENTE ISSN 0124-013}
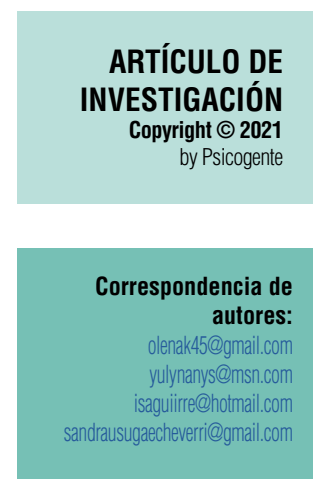

Recibido: 03-08-20 Aceptado: 07-07-21 Publicado: $30-07-21$

\section{Risco de dependência de redes sociais e Internet e sua relação com a vida e habilidades socioemocionais em uma amostra de estudantes do ensino médio do município de Envigado}

Resumen

Introducción: El continuo aumento en el uso de redes sociales e Internet, sobre todo en la población de adolescentes, requiere profundizar en el fenómeno, identificando tanto su uso problemático, como los factores relacionados con un posible riesgo de adicción.

Objetivo: Valorar el riesgo de adicción a redes sociales e Internet y su relación con habilidades para la vida y socioemocionales en una muestra de adolescentes.

Método: Un estudio cuantitativo, no experimental, de nivel correlacional, con un diseño transversal; la muestra fue compuesta por 221 estudiantes de un colegio público; se empleó la Escala de Riesgo de Adicción a las Redes Sociales e Internet (ERA-RSI), escala de Habilidades para la Vida y varias escalas para valorar las habilidades socioemocionales.

Resultados: Se identificó un nivel medio de riesgo de adicción en la muestra del estudio M 2,5 (Dt 0,6), mostrando la diferencia estadísticamente significativa en síntomas de adicción ( $p=0,001)$, uso social $(p=0,044)$ y nomofobia $(p=0,002)$ a favor del género femenino, y en rasgos frikis $(p=0,001)$ a favor del género masculino. Los puntajes entre $M 1,4(\mathrm{Dt} 0,2)$ y $M$ 2,0 (Dt 0,8$)$ indicaron un nivel bajo en las habilidades para la vida, con una diferencia significativa estadísticamente en todas las diez dimensiones constitutivas a favor del género femenino. Las habilidades socioemocionales mostraron la diferencia estadísticamente significativa en autoestima $(p=0,002)$, autoeficacia $(p=0,000)$, satisfacción vital $(p=0,001)$ y la tolerancia a la frustración $(p=0,001)$ a favor del género masculino. La resiliencia $(p=0,019)$ y las habilidades sociales $(p=0,015)$ fueron significativamente mayores en mujeres. Se identificaron las correlaciones negativas entre las variables: Total de riesgo de adicción con las habilidades para la vida $(r=-0,475 / p=0,009)$; rasgos frikis con empatía $(r=-0,140 / p=0,037)$, manejo de emociones $(r=-0,159 /$ $p=0,018)$, resiliencia $(r=-0,245 / p=0,000)$ y autoeficacia $(r=-0,238 / p=0,000)$; nomofobia con autoeficacia $(r=-0,163 / p=0,015)$ y tolerancia a la frustración $(r=-0,223 / p=0,001)$; síntomas de adicción con satisfacción vital $(r=-0,119 / p=0,008)$ y tolerancia a la frustración $(r=-0,357 / p=0,000)$; uso social de redes con el manejo de tensiones y estrés $(r=-0,169 / p=0,012)$. Igualmente, se identificaron correlaciones positivas entre el uso de redes sociales y varias dimensiones constituyentes de las habilidades sociales.

Conclusiones: Las deficiencias en las habilidades blandas de los adolescentes podrían estar relacionadas con el aumento de riesgo de adicción a redes sociales e Internet. Por otro lado, un buen desarrollo

Cómo citar este artículo (APA):

Klimenko, O., Cataño Restrepo, Y. A., Otálvaro, I. \& Úsuga Echeverri, S. J. (2021). Riesgo de adicción a redes sociales e Internet y su relación con habilidades para la vida y socioemocionales en una muestra de estudiantes de bachillerato del municipio de Envigado. Psicogente 24(46), 1-33. https://doi.org/10.17081/psico.24.46.4382 
de estas habilidades, sumado a otros factores protectores, podrían contribuir a un manejo responsable y cuidadoso del mundo virtual.

Palabras clave: riesgo de adicción a redes sociales e Internet, habilidades para la vida, adolescencia, habilidades socioemocionales.

\section{Abstract}

Introduction: Continuous increase in the use of social networks and the Internet, especially in the adolescent population, requires an in-depth study of the phenomenon, identifying its problematic use and the factors related to a possible risk of addiction.

Objective: Evaluate the risk of addiction to social networks and the Internet and its relationship with life and social-emotional skills in a sample of adolescents.

Method: A non-experimental correlational-level quantitative study was carried out, with the sample of 221 students from a public school. Was aplied the Adolescent Risk Scale for Addiction to Social Media and Internet, Life Skills Scale and some scales to assess socio-emotional skills.

Results: Was found a medium level of risk of addiction in the study sample M 2,5 (Dt 0,6), showing the statistically significant difference in addiction symptoms $(p=0,001)$, social use $(p=0,044)$ and nomophobia $(p=0,002)$ with the higher scores in the female gender, and in friki traits $(p=0,001)$ with the higher scores in the male gender. Scores between M 1,4 (Dt 0,2) and M 2,0 (Dt 0,8) indicated a low level of life skills, with a statistically significant difference in all ten constitutive dimensions with the higher scores in the female gender. The socio-emotional skills was indicated the statistically significant difference in self-esteem $(p=0,002)$, self-efficacy $(p=0,000)$, life satisfaction $(p=0,001)$ and tolerance to frustration $(p=0,001)$ with the higher scores in the male gender. Resilience $(p=0,019)$ and social skills $(p=0,015)$ were significantly higher in women. Were identified negative correlations between variables: total risk of addiction with life skills ( $r=-0,475 / p=0,009)$; friki traits with empathy $(r=-0,140 / p=0,037)$, emotion management $(r=-0,159 /$ $p=0,018)$, resilience $(r=-0,245 / p=0,000)$ and self-efficacy $(r=-0,238 / p=0,000)$; nomophobia with self-efficacy $(r=-0,163 / p=0,015)$ and frustration tolerance $(r=-0,223 / p=0,001)$; addiction symptoms with life satisfaction $(r=-0,119 / p=0,008)$ and tolerance to frustration $(r=-0,357 / p=0,000)$; social use of networks with the management of tensions and stress $(r=-0,169 / p=0,012)$. Also, positive correlations were identified between the use of social networks and some dimensions of social skills.

Conclusions: The deficiencies in the soft skills of adolescents could be related to the increased risk of adiction to social networks and the Internet. On the other hand, a good development of these skills, added to other protective factors, could contribute to a responsible and careful management of the virtual world.

Keywords: risk of addiction to social networks and the Internet, life skills, adolescence, socio-emotiona skills.

Resumo

Introdução: O aumento contínuo do uso das redes sociais e da Internet, principalmente na população adolescente, requer um aprofundamento do fenômeno, identificando tanto seu uso problemático quanto os fatores relacionados a um possível risco de dependência.

Objetivo: Avaliar o risco de dependência às redes sociais e à Internet e sua relação com habilidades de vida e socioemocionais em uma amostra de adolescentes.

Método: Estudo quantitativo, não experimental, correlacional, com desenho transversal; A amostra foi composta por 221 alunos de uma escola pública; A Rede Social e Escala de Risco de Dependência da Internet (ERA-RSI), escala de habilidades para a vida e várias escalas foram usadas para avaliar habilidades socioemocionais.

Resultados: Um nível médio de risco de dependência foi identificado na amostra do estudo M 2,5 (Dt 0,6) mostrando a diferença estatisticamente significativa nos sintomas de dependência $(p=0,001)$, uso socia $(p=0,044)$ e nomofobia ( $p=0,002)$ em favor do gênero feminino e em traços geek $(p=0,001)$ em favor do gênero masculino. Escores entre M 1,4 (Dt 0,2) e M 2,0 (Dt 0,8) indicaram baixo nível de habilidades para a vida, com diferença estatisticamente significativa em todas as dez dimensões constitutivas a favor do gênero feminino. As habilidades socioemocionais mostraram uma diferença estatisticamente significativa na autoestima $(p=0,002)$, autoeficácia $(p=0,000)$, satisfação com a vida $(p=0,001)$ e tolerância à frustração $(p=0,001)$ a favor do gênero masculino. Resiliência $(p=0,019)$ e habilidades sociais $(p=0,015)$ foram significativamente maiores nas mulheres. Foram identificadas correlações negativas entre as variáveis: risco total de dependência com habilidades para a vida $(r=-0,475 / p=0,009)$; Traços de geek com empatia $(r=-0,140$ / $p=0,037)$, gerenciamento de emoção $(r=-0,159 / p=0,018)$, resiliência $(r=-0,245 / p=0,000)$ e autoeficácia ( $r=-0,238 / p=0,000)$; Nomofobia com autoeficácia $(r=-0,163 / p=0,015)$ e tolerância à frustração $(r=-0,223$ / $p=0,001)$; sintomas de vício com satisfação com a vida $(r=-0,119 / p=0,008)$ e tolerância à frustração $(r=-0,357 / p=0,000)$; uso social de redes com o gerenciamento de tensões e estresse $(r=-0,169 / p=0,012)$. Da mesma forma, foram identificadas correlações positivas entre o uso de redes sociais e várias dimensões constituintes das habilidades sociais. 
Conclusões: As deficiências nas soft skills de adolescentes podem estar relacionadas ao aumento do risco de dependência de redes sociais e Internet. Por outro lado, um bom desenvolvimento dessas habilidades, somado a outros fatores de proteção, pode contribuir para uma gestão responsável e cuidadosa do mundo virtual.

Palavras-chave: risco de dependência de redes sociais e Internet, habilidades para a vida, adolescência habilidades socioemocionais.

\section{INTRODUCCIÓN}

En las últimas décadas, los profesionales en salud mental sostienen una polémica respecto de las adicciones no químicas, cuyo objeto representa cierto tipo de actividades y experiencias. En cuanto a estas, los autores hablan de ludopatía, incluyendo adicción a juegos virtuales, adicción al sexo, al amor romántico, a las compras, vigorexia o adicción a la imagen corporal, comida, bronceado, adicción al trabajo, y, por último, adicción a medios virtuales, como redes sociales e Internet (Pérez del Río \& Martin, 2007; Cía, 2013; Weinstein et al., 2014; Griffiths et al., 2014; Gonzales, 2015; Foster et al., 2015; Ascher \& Levounis, 2015; Petry, 2016a; Grant et al., 2016; Sussman, 2017).

En la actualidad, las adicciones químicas son ampliamente reconocidas y gozan de atención requerida debido a sus manifestaciones y consecuencias adversas, sin embargo, el tema de adicciones no químicas sigue siendo controversial, ya que todavía no hay un consenso en cuanto a los criterios para su identificación, curso clínico y tratamiento. En el DSM-5 (APA, 2013) y el CIE-10 (OMS, 1992) solo está incluida la ludopatía, incluyendo juegos virtuales. Aunque fueron consideradas varias propuestas sobre comportamientos como la adicción a Internet, sexo, compras, ejercicio físico, comer y bronceado (Petry, 2016b), no fueron incluidas finalmente, debido a la falta de estudios científicos y clínicos relacionados con estos.

En este orden de ideas, es necesario tener en cuenta que las adicciones no químicas pueden pasar desapercibidas por mucho tiempo y generar consecuencias bastante graves, por no ser detectadas o prevenidas a tiempo. Lo anterior se debe en gran parte, también, a una filosofía de la cultura contemporánea consumista, superficial e inmediatista que fomenta el consumo desaforado e innecesario, la obsesión por la imagen, la ausencia de esfuerzo e intolerancia a la frustración, permitiendo una normalización de conductas adictivas (Gonzales, 2015; Pérez del Río \& Martin, 2007).

De hecho, Becoña (2018) plantea que es necesario delimitar qué es y no es una adicción, sobre todo respecto de adicciones no químicas, ya que se corre peligro de caer en un sobrediagnóstico o una patologización innecesaria de la vida cotidiana. En este orden de ideas, en los últimos años los autores que 
consideran que muchas conductas pueden volverse adictivas, han realizado avances en la delimitación de criterios que permiten su identificación. Por ejemplo, Griffiths et al. (2014) proponen seis criterios al respecto: predominancia, modificación del estado de ánimo, tolerancia, conflicto con personas cercanas, con actividades diarias o consigo mismo, síndrome de abstinencia y recaída. Kardefelt-Winter et al. (2017) definen una adicción comportamental como una conducta altamente repetitiva que produce un daño o malestar de naturaleza perjudicial y que, a pesar de lo anterior, la persona no reduce su conducta y continúa con esta a lo largo de un tiempo significativo.

En el caso del uso de medios virtuales, estos se han vuelto completamente indispensables para la vida en el siglo XXI, siendo las personas que no tienen acceso, afectadas de una u otra forma en su desempeño académico, laboral, entre otros. Desde este punto de vista, los medios virtuales pueden, igualmente, ser un objeto cultural en relación al cual se genere un comportamiento adictivo, sobre todo en poblaciones de mayor uso, como los adolescentes contemporáneos, nativos digitales que crecieron empleando estos medios desde edades tempranas.

Al respecto de lo anterior, en la literatura se encuentran ya bastantes estudios que expresan la preocupación en el tema, incluyendo la creación de instrumentos que permiten identificar la presencia de comportamiento adictivo en relación al uso de medios virtuales, donde se incluyen las redes sociales e Internet (Chóliz \& Villanueva, 2011; Basteiro et al., 2013; Escurra \& Salas, 2014; Peris et al., 2018).

Sin embargo, el tema de adicción a medios virtuales, redes sociales e Internet sigue siendo todavía un objeto de controversia entre los autores (Caro, 2017). Hay algunos que afirman que redes sociales en línea no son adictivas, ya que hacen parte integral e indispensable de la vida de adolescentes y jóvenes contemporáneos, y aunque pueden producir cierto tipo de problemáticas, la adicción no es una de estas (Fernández, 2013; Carbonell \& Oberst, 2015).

Algunos consideran que la adicción al Internet no es un trastorno en sí mismo sino un problema comórbido a trastornos de base como: depresión, trastorno bipolar, trastorno de conducta alimentaria, control de impulsos, pobre regulación emocional, alcoholismo o personalidad antisocial, siendo expresión y enmascaramiento de estos (Huang \& Alessi, 1997; Shapira et al., 1998; Shapira et al., 2000; Hormes et al., 2014). En esta línea se encuentran estudios que identifican la presencia de diversas problemáticas en personas con riesgo de abuso de medios virtuales, por ejemplo, sintomatología 
depresiva y ansiógena y baja autoestima, al igual como problemas escolares (Flores et al., 2013).

Por otro lado, hay autores que se inclinan a considerar la adicción a medios virtuales como un problema primario, siendo una variante de posibles trastornos de comportamiento adictivo (Sánchez-Carbonell et al., 2008; Echeburúa \& De Corral, 2010; Fuertes \& Armas, 2018).

En este aspecto, Arias et al. (2012) afirman que hay dos clases de adicción a nuevas tecnologías: pasiva, producida, por ejemplo, por televisión, que incide en el aislamiento social, dejándose el sujeto influenciar por sus contenidos; y activa, siendo una forma más grave con problemáticas de índole social, económica y psicológica asociadas, y se expresa en adicción a sexo, juego, redes sociales, compras, etc., empleando medios virtuales. Davis (2001) habla de adicción a Internet generalizada, que hace referencia al uso excesivo a nivel de muchas áreas sin focalizarse en alguna aplicación en particular; y específica, que representa enfoque en una concreta, como: videojuegos, redes sociales, apuestas, cibersexo, compras virtuales, etc.

Ferre et al. (2016) plantean que para identificar la presencia de un comportamiento adictivo en caso del uso de medios virtuales se debe aplicar tres criterios claros que definen también los trastornos por consumo de sustancias: tipo problemático de uso, que continúe incluso cuando hay efectos negativos significativos en varias áreas de la vida y un deterioro o malestar durante por lo menos 12 meses; tolerancia y abstinencia. Estos autores consideran que actualmente se está en evolución del concepto de adicción comportamental, y recomiendan la inclusión de las adicciones tanto a sustancias como sin sustancias al espectro de trastornos impulsivo-compulsivos, siendo ambos tipos de comportamiento adictivo equiparables en muchos aspectos como historia, mecanismos neurobiológicos, respuesta al tratamiento, tolerancia, entre otros.

Algunos autores dirigen la atención específicamente a la adicción a las redes sociales que se ha incrementado en los últimos años, al mismo tiempo que los estudios relacionados con este fenómeno siguen siendo relativamente escasos, lo cual indica la importancia de indagar por los motivos de uso, examinar sus consecuencias negativas y explorar el riesgo de adicción a estas en distintas poblaciones (Griffiths et al., 2014).

En cuanto a la prevalencia de adicción a medios virtuales es igualmente controversial, debido a la ausencia de un consenso en criterios diagnósticos. 
Durkee et al. (2012) realizaron un estudio con 11.356 adolescentes de 11 países europeos, identificando una prevalencia de uso patológico de Internet en torno a 3,5 \% de la población, y el uso adaptativo asociado a consecuencias menores negativas en $13,5 \%$, siendo el sexo masculino con mayor grado de manifestación de esta problemática. En relación al abuso del móvil, Mok et al. (2014) refieren que alrededor de 17,9 \% de adolescentes coreanos presentan adicción a smartphone, y, además, $24 \%$ de estos requirió en algún momento un ingreso hospitalario. Casi un $3 \%$ de la población española de 15 a 64 tiene uso compulsivo de Internet, llegando a 9,5\% en la edad entre 15 y 24 años (Observatorio Español de las Drogas y las Adicciones, 2019). A nivel mundial se indica entre un 1,58 y un 2 \% de adicción al móvil (Chóliz, 2010), cifra que ha aumentado en los últimos años debido al uso de WhatsApp, ascendiendo a $11 \%$ en adolescentes entre 11 y 14 años en la población europea (López- Fernández et al., 2014). La preocupación sobre el problema se ha manifestado en varios países, sobre todo con mayor desarrollo tecnológico, como Japón, por ejemplo, y se han creado centros de tratamiento para el problema en Alemania, Reino Unido y Estados Unidos, entre otros. Igualmente, los autores resaltan que este tipo de comportamiento adictivo al respecto de medios tecnológicos afecta con mayor frecuencia a las edades más jóvenes como adolescentes (Arias et al., 2012).

Por otro lado, también se pueden encontrar estudios que no reportan la presencia de casos significativos de adicción a Internet en algunas poblaciones de adolescentes, como un estudio realizado por Ruiz-Palmero et al. (2016) en Málaga. Al mismo tiempo, se pueden observar estudios que indican el beneficio de medios tecnológicos para los procesos de aprendizaje de adolescentes. En este aspecto, muestran una percepción positiva sobre el aporte que pueden ofrecer los medios virtuales para el proceso de estudio, interacción con los pares y relaciones interpersonales (Pérez, 2016).

Lo anterior muestra que el elemento importante en este problema de adicción a los medios virtuales, las redes sociales e Internet, al igual que en relación a otro tipo de comportamiento adictivo, es la prevención que permite no solo concientizar sobre su uso adecuado, sino también fortalecer los recursos a nivel psicosocial con los cuales cuentan los adolescentes con el fin de que puedan tomar decisiones adecuadas y autogesionar de forma sana su propio comportamiento, apoyados en redes de soporte social.

En este orden de ideas, tanto un alto porcentaje de presencia de comportamiento adictivo y su exponencial crecimiento, como la necesidad del desarrollo de estrategias preventivas funcionales, llevan a preguntarse por 
los factores de riesgo relacionados con el fenómeno del comportamiento adictivo.

Los avances en neurociencia y biología molecular han permitido acercarse a una mejor comprensión de las bases neurobiológicas del fenómeno de adicción. Estos avances científicos impulsaron el surgimiento de un modelo explicativo de adicciones como una "enfermedad cerebral", afirmando que el uso de sustancias modifica la estructural, el funcionamiento y la química del cerebro (Riva-Posse, 2016).

Los estudios relacionados con la bioquímica de nuestro organismo sugieren que ésta puede ser un factor importante en el surgimiento de un comportamiento adictivo, cuyo objeto puede ser tanto químico, como no químico (Pert, 1986). De hecho, hay estudios que indican la relación entre la adicción a sustancias psicoactivas con otros comportamientos adictivos como el juego patológico, uso excesivo de Internet y videojuegos, siendo el comportamiento adictivo una estructura comportamental de sujetos evaluados (Pedrero, 2010). Lo anterior pasa desapercibido a menudo, debido precisamente a una mayor atención y relevancia social de adicciones químicas consideradas inadmisibles. Los estudios indican incluso que el abandono de ingesta de una sustancia psicoactiva lleva al aumento de la ingesta de otras sustancias o a una exacerbación de otro tipo de conductas no relacionadas con sustancias químicas, las cuales podrían o no estar en el repertorio de conductas previamente asociadas con la adicción principal que se pretende extinguir (Magura \& Rosenblum, 2000; Sánchez \& Rodríguez, 2006; Pedrero, 2010). Al respecto algunos estudios indican que las bases neuronales subyacentes, tanto a las adicciones químicas, como no químicas son las mismas (Han et al., 2007; Kiefer et al., 2017).

Por otro lado, es necesario considerar que, aunque las bases neurobiológicas son más o menos iguales en todos los seres humanos, y que cualquier sustancia que altera el funcionamiento de nuestro sistema nervioso produce un efecto aproximadamente similar en distintos seres humanos, sin embargo, los estudios indican que solo un porcentaje de la población expuesta al consumo de estas sustancias desarrolla un comportamiento adictivo (Méndez-Díaz et al., 2017). Lo anterior también se aplica a las adicciones no químicas: todos los seres humanos realizan actividades que se relacionan con las más frecuentes, pero solo un porcentaje desarrolla un comportamiento adictivo en relación a estas. 
Según Becoña (2016), la biología, siendo un factor de mucha relevancia en las adicciones, no puede considerarse como una aproximación explicativa exclusiva de las mismas. Es necesario un abordaje biopsicosocial que incluye la consideración de la participación de los factores psicosociales en la problemática.

En este orden de ideas, es importante orientar la atención, sobre todo, a los recursos personológicos como elementos clave para comprender el fenómeno de comportamiento adictivo, y especialmente, en caso de adicciones no químicas, al igual como factores de índole social, donde la influencia de la filosofía social predominante de consumo desaforado, con los ideales superfluos de disfrute inmediato, exhibicionismo individualista y presión social hacia el conformismo puede incidir de forma significativa en surgimiento de conductas adictivas en personas de mayor vulnerabilidad psicológica, como un camino en la búsqueda de su propia identidad (Ovejero, 2000; Riva-Posse, 2016).

Los autores están de acuerdo que el comportamiento adictivo es el resultado de varios factores confluyentes, tanto biológicos, como ambientales, entre los cuales están pautas de crianza, situación social del desarrollo, características personológicas y habilidades, estrategias de afrontamiento, trastornos afectivos, entre otros (Méndez-Díaz et al., 2017; González-López \& NaranjoFundora, 2013; Mendoza \& Vargas, 2017; Pons, 2008).

Los recursos personológicos, tanto cognitivos, como socioemocionales, han sido resaltados por varios autores como elementos de gran peso en la capacidad de una persona de autogestionar su comportamiento de forma autónoma y hacer frente a la posibilidad de desarrollar un comportamiento adictivo (Becoña \& Oblitas, 2003).

Siendo adicciones no químicas todavía un tema en estudio, es importante indagar por estos recursos a nivel personal que podrían actuar como factores protectores, sobre todo en edades de mayor vulnerabilidad en cuanto a la emergencia de comportamiento aditivo, como la adolescencia. En ese orden de ideas, este estudio orienta su atención a la presencia del riesgo de adicción a las redes sociales e Internet en una muestra de estudiantes de secundaria y su relación con las habilidades para la vida, como un conjunto de recursos psicológicos tanto cognitivos como emocionales y motivacionales, de gran relevancia, que permiten enfrentar distintas situaciones vitales, tomar decisiones más funcionales, y administrar de mejor forma las emociones y relaciones interpersonales. Igualmente, se consideraron otras caracterís- 
ticas socioemocionales como resiliencia, autoestima, autoeficacia, satisfacción vital, tolerancia a la frustración, planificación y toma de decisiones, empatía, manejo de emociones, apego a iguales y habilidades sociales. Para la valoración de habilidades para la vida en este estudio se empleó el modelo propuesto por OMS (1999), que ha sido ampliamente utilizado en tema de estrategias de prevención de consumo de sustancias, específicamente, en la población de adolescentes.

\section{MÉTODO}

\subsection{Tipo de estudio}

Investigación de enfoque cuantitativo, no experimental de nivel correlacional, con un diseño transversal.

\subsection{Participantes}

Para el estudio se seleccionó uno de los colegios públicos del municipio de Envigado, que cuenta con la mayor cantidad de estudiantes. Para la muestra se escogió al azar un grupo de cada grado de bachillerato desde el 7 hasta el 11. Después de descartar instrumentos incompletos, la muestra quedó compuesta por 221 estudiantes. El promedio de la edad de la muestra fue de $M=15,6$ (Dt. 1,5). 52,9 \% del total fueron hombres, $46,6 \%$, mujeres y $0,5 \%$ indicaron otro género. El estrato socioeconómico predominante fue el $3(64,7 \%)$. Más de la mitad de los estudiantes vive con ambos padres $(51,1 \%)$, seguidos por la familia monoparental con jefatura femenina (42,5\%). El $95 \%$ de la muestra cuenta con un celular propio, aproximadamente la mitad tiene un computador propio $(57,5 \%)$ y $93,2 \%$, el Internet en su casa.

\subsection{Instrumentos}

Para la recolección de información se empelaron varios instrumentos que se describen a continuación:

Para la medición de la variable del riesgo de adicción a redes sociales e Internet se empleó la Escala de Riesgo de Adicción-Adolescente a las Redes Sociales e Internet (ERA-RSI), elaborada y validada por Peris et al. (2018) en la población de adolescentes españoles. Este instrumento representa una escala Likert con cuatro opciones de respuesta. La escala mide cuatro factores: síntomas-adicción, uso-social, rasgos-frikis y nomofobia, que explican el 46,48 \% de la varianza. Los autores reportan una buena consistencia interna de la escala $(\alpha=0,90)$, y los valores de test retest fueron entre $r=0,76$ y $r=0,88$. 
Para la variable de habilidades para la vida se utilizó el Test de Habilidades para la Vida, diseñado por Díaz et al. (2013) y validado en la población colombiana. Representa una escala Likert con cinco opciones de respuesta y evalúa diez dimensiones de habilidades para la vida, según el modelo teórico propuesto por la OMS (1999): Conocimiento de sí mismo, Empatía, Comunicación efectiva y asertiva, Relaciones interpersonales, Toma de decisiones, Solución de problemas y conflictos, Pensamiento creativo, Pensamiento crítico, Manejo de sentimientos y emociones, Manejo de tensiones y estrés. Los autores reportan una satisfactoria confiabilidad del instrumento con coeficientes de alfa Cronbach que oscilan entre 0,60 y 0,85 en los diez factores.

Para medir las habilidades socioemocionales en los adolescentes de la muestra se emplearon las siguientes escalas:

Para valorar la resiliencia se utilizó el Cuestionario de Resiliencia para Niños y Adolescentes, diseñado por González (2016) y validado en la población mexicana, consta de 32 ítems, con buena consistencia interna y alfa de Cronbach $=0,91$.

Para evaluar el autoestima se empleó la Escala de Autoestima para Adolescentes, diseñada por Rosenberg (1965), es una escala unidimensional compuesta de diez ítems y reporta alfa Cronbach de 0,92 en la población española (Oliva et al., 2011) y de 0,96 en la población latinoamericana (Martínez \& Alfaro, 2019).

La variable de autoeficacia evaluada con la Escala de Autoeficacia Generalizada, diseñada por Baessler y Schwarzer (1996), representa una escala unidimensional de diez ítems, ha mostrado alfa Cronbach de 0,82 para la población española (Oliva et al., 2011) y de 0,74 en la población colombiana (Escobar \& Zambrano, 2015).

Para la variable de satisfacción vital se empleó la Escala para la Evaluación de la Satisfacción Vital, diseñada por Huebner (1991), escala unidimensional de siete ítems, con el coeficiente de fiabilidad en la población española de $\alpha=0,81$ (Oliva et al., 2011) y para la población latinoamericana de $\alpha=0,86$ (Alfaro et al., 2016).

La variable de tolerancia a la frustración se valoró con la Escala para la Evaluación de la Tolerancia a la Frustración, representa adaptación española de la subescala Stress Management of Emotional Quotient Inventory (EQ-i, 
YV) de Bar-On \& Parker (2000), consta de 8 items, evalúa la percepción de los niños/as y adolescentes sobre su propia capacidad para el manejo del estrés-tolerancia al estrés y control impulsivo, muestra un índice de alfa de Cronbach de 0,77 para la población española (Oliva et al., 2011).

Para medir la variable de apego a los iguales se empleó la Escala para la Evaluación del Apego a Iguales, de Amrsden \& Greemberg (1987), consta de 21 items, compuesta por tres subescalas de confianza, comunicación y alienación, cuya validación en la población española mostró una fiabilidad de alfa de Cronbach de 0,73 (Oliva et al., 2011).

Y, finalmente, la variable de habilidades sociales se evaluó con la Escala para la Evaluación de las Habilidades Sociales, diseñada y validada por Oliva et al. (2011), consta de 12 ítems que permiten valorar la percepción de los adolescentes sobre sus habilidades sociales comunicativas o relacionales, la asertividad (positiva y negativa) y las habilidades de resolución de conflictos, cuyo índice de consistencia interna en la población española fue de 0,69.

\subsection{Procedimiento}

Para el proceso de recolección de información, inicialmente se procedió a solicitar el permiso de los directivos del colegio y la respectiva programación del proceso de envío de consentimientos informados a los padres de familia de cada grupo participante. Se realizó la aplicación de los cuestionarios durante las jornadas escolares con el respaldo y seguimiento de la dirección académica y los investigadores. Se respetó la participación voluntaria de estudiantes en cada grupo escogido.

\subsection{Consideraciones éticas}

El estudio se realizó bajo las regulaciones establecidas en la Ley 1090 de 2006 del Colegio Colombiano de Psicólogos, y la Resolución 8430 de 1993 del Ministerio de Salud, que reglamentan las normas administrativas, científicas y técnicas para las investigaciones en salud. La presente investigación se clasifica como un estudio de riesgo mínimo. Todos los participantes fueron informados sobre sus objetivos y procedimientos y se firmó el consentimiento informado con los acudientes de los participantes, siendo más de $90 \%$ de la muestra menores de edad.

\subsection{Análisis de datos}

La prueba de distribución de variables del estudio Kolmogorov-Smirnov indico la distribución no paramétrica para todas las variables. Se utilizaron 
la estadística descriptiva para estimar valores de las variables, la prueba no paramétrica U de Mann Whitney para la comparación de dos grupos y Chi cuadrado en caso de más de dos grupos, también se empleó el coeficiente de correlación de Spearman para hallar la correlación entre las variables de los instrumentos aplicados. La significancia estadística se estableció con un valor $p<0,05$. Los datos fueron procesados con el paquete estadístico SPSS versión 22 para Windows. Para el análisis de diferencia de riesgo de adicción según edad se recodificó la variable de edad en dos: adolescencia temprana (12-14) y adolescencia tardía (15-18).

\section{RESULTADOS}

En cuanto a las habilidades para la vida en los estudiantes de la muestra, se identificaron valores muy bajos en las diez dimensiones correspondientes. En la escala de 1 a 5 los valores de variables fueron entre M 1,4 (Dt 0,2) y M 2,0 (Dt 0,8), indicando dificultades en la muestra del estudio en cuanto al manejo de las habilidades para la vida. Los menores valores se obtuvieron en las variables de toma de decisiones (M 1,4/Dt 0,2), y solución de problemas y conflictos ( $M$ 1,4/Dt 0,2), seguido por empatía (M1,5/Dt 0,2), pensamiento creativo y crítico (M 1,5/Dt 0,2) y manejo de sentimientos y emociones (M 1,5/Dt 0,2). Las variables de conocimiento de sí mismo ( $M$ 1,6/Dt 0,5), comunicación efectiva y asertiva (M 1,6/ Dt 0,2) y relaciones interpersonales (M 1,6/Dt 0,2) mostraron una puntuación un poco mayor que el resto de las variables, siendo la variable de manejo de tensiones y estrés la que obtuvo el mayor puntaje (M 2,0/Dt 0,8).

Tabla 1

Valores de dimensiones de habilidades para la vida en la muestra del estudio

\begin{tabular}{cc}
\hline VARIABLES & M (DT) \\
\hline Conocimiento de sí mismo & $1,6(0,5)$ \\
Empatía & $1,5(0,2)$ \\
Comunicación efectiva y asertiva & $1,6(0,2)$ \\
Relaciones interpersonales & $1,6(0,2)$ \\
Toma decisiones & $1,4(0,2)$ \\
Solución de problemas y conflictos & $1,4(0,2)$ \\
Pensamiento creativo & $1,5(0,2)$ \\
Pensamiento crítico & $1,5(0,2)$ \\
Manejo de sentimientos y emociones & $1,5(0,2)$ \\
Manejo de tensiones y estrés & $2,0(0,8)$ \\
\hline
\end{tabular}

La comparación de las habilidades para la vida según la variable de género, mostró estadísticamente una diferencia significativa en todas las diez dimensiones constitutivas a favor del género femenino. 
Tabla 2

Diferencias de las habilidades para la vida según la variable de género

\begin{tabular}{ccccc}
\hline VARIABLES & $\begin{array}{c}\text { MASCULINO } \\
\text { ME(RI) }\end{array}$ & $\begin{array}{c}\text { FEMENINO } \\
\text { ME(RI) }\end{array}$ & U DE MANN-WHITNEY & P \\
\hline Conocimiento de sí mismo & $1,4(0,2)$ & $1,6(0,3)$ & 3777,500 & 0,000 \\
Empatía & $1,4(0,2)$ & $1,6(0,3)$ & 3575,000 & 0,000 \\
Comunicación efectiva y asertiva & $1,5(0,2)$ & $1,6(0,3)$ & 3575,000 & 0,000 \\
Relaciones interpersonales & $1,5(0,3)$ & $1,6(0,4)$ & 4786,000 & 0,008 \\
Toma decisiones & $1,3(0,3)$ & $1,4(0,3)$ & 5091,000 & 0,047 \\
Solución de problemas y conflictos & $1,3(0,2)$ & $1,5(0,3)$ & 4966,000 & 0,024 \\
Pensamiento creativo & $1,4(0,3)$ & $1,5(0,4)$ & 4949,000 & 0,022 \\
Pensamiento crítico & $1,4(0,3)$ & $1,5(0,3)$ & 4662,000 & 0,004 \\
Manejo de sentimientos y emociones & $1,4(0,2)$ & $1,6(0,3)$ & 3575,000 & 0,000 \\
Manejo de tensiones y estrés & $1,5(1,2)$ & $1,8(0,5)$ & 4953,500 & 0,021 \\
\hline
\end{tabular}

En relación con las variables de las habilidades socioemocionales en la muestra del estudio, se identificó un nivel medio alto en el apego a los iguales (M 5,4 (Dt 0,8) en la escala de 1 a 7).

El resto de las variables se ubicaron en los niveles medios y bajos. Se observó una baja puntuación en la resiliencia ( $\mathrm{M} \mathrm{2,2}$ (Dt 0,4) en la escala de 1 a 5), autoeficacia (M 2,7 (Dt 0,6) en la escala de 1 a 7), las habilidades sociales ( $\mathrm{M} 2,5(0,8)$ en la escala de 1 a 7). En cuanto al autoestima ( 2 (Dt 0,5) en la escala de 1 a 4), satisfacción vital (M 3,2 (Dt 1,2) en la escala de 1 a 7) y tolerancia a la frustración M 3,4 (Dt 0,9), se identificaron niveles medio-bajos en la puntuación.

Tabla 3

Valores de variables de habilidades socioemocionales en la muestra del estudio

\begin{tabular}{cc}
\hline VARIABLES & M (DT) \\
\hline Resiliencia & $2,2(0,4)$ \\
Autoestima & $2,0(0,5)$ \\
Autoeficacia & $2,7(0,6)$ \\
Satisfacción vital & $3,2(1,2)$ \\
Tolerancia a la frustración & $3,4(0,9)$ \\
Apego a los iguales & $5,4(0,8)$ \\
Total habilidades sociales & $2,5(0,8)$ \\
\hline
\end{tabular}

La comparación de las variables de las habilidades socioemocionales según el género, estadísticamente indicó la diferencia significativa en autoestima $(p=0,002)$, autoeficacia $(p=0,000)$, satisfacción vital $(p=0,001)$ y la tolerancia a la frustración $(p=0,001)$ a favor del género masculino. La resiliencia $(p=0,019)$ y las habilidades sociales $(p=0,015)$ fueron significativamente mayores en 
mujeres. En cuanto al apego a los iguales no se identificó la diferencia entre los géneros en la muestra del estudio $(p=0,379)$.

Tabla 4

Diferencias de las habilidades socioemocionales según género

\begin{tabular}{ccccc}
\hline VARIABLES & MASCULINO ME(RI) & FEMENINO ME(RI) & U DE MANN-WHITNEY & P \\
\hline Resiliencia & $2,1(0,5)$ & $2,4(0,7)$ & 4921,000 & 0,019 \\
Autoestima & $2,2(0,7)$ & $2,1(1)$ & 4559,500 & 0,002 \\
Autoeficacia & $2,8(0,8)$ & $2,4(0,9)$ & 3778,500 & 0,000 \\
Satisfacción vital & $3,5(1,6)$ & $3,2(1,9)$ & 4406,000 & 0,001 \\
Tolerancia a la frustración & $3,6(1,1)$ & $3,1(1,1)$ & 4388,500 & 0,001 \\
Apego a los iguales & $5,4(1)$ & $5,5(0,9)$ & 5611,500 & 0,379 \\
Total habilidades sociales & $2,2(1)$ & $2,6(1,1)$ & 5625,500 & 0,015 \\
\hline
\end{tabular}

En relación al riesgo de adicción a redes sociales e Internet, se encontraron niveles medios en las variables de síntomas de adicción (M 2,6 (Dt 0,4), uso social (M 2,5 (Dt 0,6), nomofobia (M 2,4 (Dt 0,7) y en total de riesgo de adicción (M 2,5 (Dt 0,6) en la escala de 1 a 4. En la variable de rasgos frikis se obtuvo un nivel medio bajo (M 1,8 (Dt 0,6).

Tabla 5

Valores de variables en riesgo de adicción a redes sociales e Internet

\begin{tabular}{cc}
\hline VARIABLES & M(DT) \\
\hline Síntomas adicción & $2,6(0,4)$ \\
Uso social & $2,5(0,6)$ \\
Rasgos frikis & $1,8(0,6)$ \\
Nomofobia & $2,4(0,7)$ \\
Total riesgo adicción & $2,5(0,6)$ \\
\hline
\end{tabular}

En cuanto a la comparación de riesgo de adicción a redes sociales e Internet según el género, se identificó la diferencia estadísticamente significativa en síntomas de adicción $(p=0,001)$, uso social $(p=0,044)$ y nomofobia $(p=0,002)$ a favor del género femenino, y en rasgos frikis $(p=0,001)$ a favor del género masculino. El total de riesgo de adicción $(p=0,091)$ no indicó diferencia significativa entre los géneros.

Tabla 6

Diferencias en riesgo de adicción a redes sociales e Internet según el género

\begin{tabular}{ccccc}
\hline VARIABLES & MASCULINO ME(RI) & FEMENINO ME(RI) & U DE MANN-WHITNEY & P \\
\hline Síntomas adicción & $2,2(0,7)$ & $2,4(0,6)$ & 4497,500 & 0,001 \\
Uso social & $2,2(0,7)$ & $2,4(1,2)$ & 5078,000 & 0,044 \\
Rasgos frikis & $1,8(0,8)$ & $1,5(1,1)$ & 4469,000 & 0,001 \\
Nomofobia & $2,1(1)$ & $2,5(1,2)$ & 4552,000 & 0,002 \\
Total riesgo adicción & $2,1(0,5)$ & $2,2(0,7)$ & 5230,500 & 0,091 \\
\hline
\end{tabular}


En cuanto al riesgo de adicción a redes sociales e Internet según el rango de edad, estadísticamente no se identificó la diferencia significativa entre los grupos de adolescencia temprana y tardía.

\section{Tabla 7}

Diferencias en riesgo de adicción a redes sociales e Internet según el rango de edad

\begin{tabular}{ccccc}
\hline VARIABLES & $\begin{array}{c}\text { ADOL. TEMPRANA } \\
\text { ME(RI) }\end{array}$ & $\begin{array}{c}\text { ADOL. TARDÍA } \\
\text { ME(RI) }\end{array}$ & $\begin{array}{c}\text { U DE } \\
\text { MANN-WHITNEY }\end{array}$ & P \\
\hline Síntomas adicción & $2,3(0,6)$ & $2,3(0,5)$ & 6010,500 & 0,852 \\
Uso social & $2,4(0,8)$ & $2,3(0,8)$ & 6029,500 & 0,883 \\
Rasgos frikis & $1,6(1)$ & $1,8(0,9)$ & 5729,500 & 0,435 \\
Nomofobia & $2,1(1)$ & $2,4(1,4)$ & 5475,000 & 0,188 \\
Total riesgo adicción & $2,1(0,8)$ & $2,1(0,7)$ & 5800,500 & 0,530 \\
\hline
\end{tabular}

La comparación del riesgo de adicción a redes sociales e Internet según las variables de tenencia de celular, computador y acceso a Internet, se identificó la diferencia significativa en la variable de uso social $(p=0,022)$ a favor del grupo que cuenta con el celular propio, en la variable de rasgos frikis $(p=0,009)$ a favor del grupo que tiene el computador propio, y en síntomas de adicción $(p=0,044)$, rasgos frikis $(p=0,004)$ y total de riesgo de adicción $(p=0,012)$ a favor del grupo que no cuenta con el acceso a Internet en su lugar de vivienda.

\section{Tabla 8}

Diferencias en riesgo de adicción a redes sociales e Internet según la tenencia del celular, computador e Internet

\begin{tabular}{|c|c|c|c|c|}
\hline VARIABLES & $\begin{array}{l}\text { TIENE CELULAR } \\
\text { ME(RI) }\end{array}$ & $\begin{array}{c}\text { NO TIENE CELULAR } \\
\text { ME(RI) }\end{array}$ & $\begin{array}{c}\text { U DE } \\
\text { MANN-WHITNEY }\end{array}$ & $\mathbf{P}$ \\
\hline Síntomas adicción & $2,3(0,6)$ & $2,4(0,9)$ & 990,000 & 0,424 \\
\hline Uso social & $2,3(0,8)$ & $1,8(0,8)$ & 681,500 & 0,022 \\
\hline Rasgos frikis & $1,8(1)$ & $1,6(1)$ & 1001,000 & 0,454 \\
\hline Nomofobia & $2,3(1,2)$ & $2,1(0,7)$ & 878,500 & 0,180 \\
\hline \multirow[t]{2}{*}{ Total riesgo adicción } & $2,1(0,7)$ & $2(0,5)$ & 829,500 & 0,115 \\
\hline & $\begin{array}{l}\text { TIENE COMPUTADOR } \\
\text { ME(RI) }\end{array}$ & $\begin{array}{c}\text { NO TIENE COMPUTADOR } \\
\text { ME(RI) }\end{array}$ & $\begin{array}{c}\text { U DE } \\
\text { MANN-WHITNEY }\end{array}$ & $\mathbf{P}$ \\
\hline Síntomas adicción & $2,3(0,6)$ & $2,3(0,6)$ & 5862,500 & 0,820 \\
\hline Uso social & $2,3(1)$ & $2,3(0,8)$ & 5615,000 & 0,450 \\
\hline Rasgos frikis & $1,9(1)$ & $1,6(0,8)$ & 5197,000 & 0,009 \\
\hline Nomofobia & $2,3(1)$ & $2,5(1,1)$ & 5406,000 & 0,230 \\
\hline \multirow[t]{2}{*}{ Total riesgo adicción } & $2,1(0,7)$ & $2,1(0,6)$ & 5933,000 & 0,939 \\
\hline & $\begin{array}{l}\text { TIENE INTERNET } \\
\text { ME(RI) }\end{array}$ & $\begin{array}{c}\text { NO TIENE INTERNET } \\
\text { ME(RI) }\end{array}$ & $\begin{array}{c}\text { U DE } \\
\text { MANN-WHITNEY }\end{array}$ & $\mathbf{P}$ \\
\hline Síntomas adicción & $2,3(0,6)$ & $2,6(0,6)$ & 1064,000 & 0,044 \\
\hline Uso social & $2,3(0,7)$ & $2,6(2)$ & 1207,000 & 0,157 \\
\hline Rasgos frikis & $1,8(0,8)$ & $2,3(0,5)$ & 862,500 & 0,004 \\
\hline Nomofobia & $2,3(1,2)$ & $2,6(0,8)$ & 1186,500 & 0,133 \\
\hline
\end{tabular}


En cuanto a la diferencia en el riesgo de adicción a redes sociales e Internet según el tipo de familia de los participantes, se identificó la diferencia estadísticamente significativa solo en la variable de uso social $(p=0,024)$, indicando el mayor promedio en el grupo que vive con abuelos $M 2,6$ (Dt 0,9$)$, seguido por el grupo que vive con la madre $M 2,4($ Dt 0,8$)$ y con el menor promedio en el grupo que vive con ambos padres M2,1 (Dt 0,9).

Tabla 9

Diferencias en riesgo de adicción a redes sociales e Internet según tipo de familia

\begin{tabular}{ccccccc}
\hline VARIABLES & $\begin{array}{c}\text { AMBOS } \\
\text { ME(RI) }\end{array}$ & $\begin{array}{c}\text { MADRE } \\
\text { ME(RI) }\end{array}$ & $\begin{array}{c}\text { PADRE } \\
\text { ME(RI) }\end{array}$ & $\begin{array}{c}\text { ABUELOS } \\
\text { ME(RI) }\end{array}$ & $\begin{array}{c}\text { CHI } \\
\text { CUADRADO }\end{array}$ & P \\
\hline Síntomas adicción & $2,3(0,6)$ & $2,4(0,5)$ & $2,1(0,5)$ & $2,2(0,7)$ & 4,780 & 0,189 \\
Uso social & $2,1(0,9)$ & $2,4(0,8)$ & $2,3(0,9)$ & $2,6(0,9)$ & 9,395 & 0,024 \\
Rasgos frikis & $1,6(0,8)$ & $1,8(1,2)$ & $1,8(0,6)$ & $1,6(0,9)$ & 5,365 & 0,147 \\
Nomofobia & $2,3(1)$ & $2,3(1,4)$ & $2,3(1)$ & $2,5(1,3)$ & 0,369 & 0,947 \\
Total riesgo adicción & $2,1(0,7)$ & $2,2(0,7)$ & $2,1(0,7)$ & $2,2(0,3)$ & 4,693 & 0,196 \\
\hline
\end{tabular}

Al respecto de correlaciones entre variables de riesgo de adicción a redes sociales y las habilidades para la vida, se identificaron varias correlaciones que, a pesar de ser bajas en su mayoría, fueron significativas.

En primer lugar, se observó una correlación mediana, negativa y significativa entre total de riesgo de adicción y total de habilidades para la vida $(r=-0,475$, $p=0,009)$.

La variable de rasgos frikis mostró una correlación baja, negativa y significativa con la variable de empatía $(r=-0,140, p=0,037)$ y con la variable de manejo de emociones $(r=-0,159, p=0,018)$.

La variable de uso social de redes sociales fue la que más correlaciones obtuvo con las distintas dimensiones de las habilidades para la vida. En este aspecto, la dimensión de manejo de tensiones y estrés mostró una correlación negativa, baja y significativa $(r=-0,169, p=0,012)$ con el uso social de redes sociales.

Las demás correlaciones fueron positivas, bajas y significativas. En este aspecto se identificaron las correlaciones entre la variable uso social de redes sociales y conocimiento de sí mismo $(r=0,214, p=0,001)$, empatía $(r=0,223$, $p=0,001)$, comunicación efectiva y asertiva $(r=0,223, p=0,001)$, relaciones interpersonales $(r=0,204, p=0,001)$, toma de decisiones $(r=0,200, p=0,003)$, solución de problemas y conflictos $(r=0,178, p=0,008)$, pensamiento creativo 
$(r=0,186, p=0,006)$, pensamiento crítico $(r=0,196, p=0,004)$, manejo de sentimientos y emociones $(r=0,223, p=0,001)$.

En cuanto a la correlación entre variables de riesgo de adicción y otras variables socioemocionales, se identificaron las correlaciones negativas, bajas y significativas en cuanto a: rasgos frikis con resiliencia $(r=-0,245, p=0,000)$ y autoeficacia $(r=-0,238, p=0,000)$; nomofobia con autoeficacia $(r=-0,163, p=0,015)$ y tolerancia a la frustración $(r=-0,223, p=0,001)$; síntomas de adicción con satisfacción vital $(r=-0,119, p=0,008)$ y tolerancia a la frustración $(r=-0,357$, $p=0,001)$.

Tabla 10

Correlación entre variable de riesgo de adicción a redes sociales e Internet y variables de habilidades socioemocionales

\begin{tabular}{ccc}
\hline VARIABLES & RHO SPEARMAN & P \\
\hline Conocimiento de sí mismo/ uso social & $0,214^{* *}$ & 0,001 \\
Empatía/uso social & $0,223^{* *}$ & 0,001 \\
Empatí/rasgos frikis & $-0,140^{*}$ & 0,037 \\
Comunicación efectiva y asertiva/uso social & $0,223^{* *}$ & 0,001 \\
Relaciones interpersonales/uso social & $0,204^{* *}$ & 0,001 \\
Toma de decisiones/uso social & $0,200^{* *}$ & 0,003 \\
Solución de problemas y conflictos/uso social & $0,178^{* *}$ & 0,008 \\
Pensamiento creativo/uso social & $0,196^{* *}$ & 0,006 \\
Pensamiento crítico/uso social & $0,195^{* *}$ & 0,004 \\
Manejo de sentimientos y emociones/uso social & $0,223^{* *}$ & 0,001 \\
Manejo de emociones/rasgos frikis & $-0,159^{*}$ & 0,018 \\
Manejo de tensiones y estrés/uso social & $-0,169^{*}$ & 0,012 \\
Total riesgo de adicción/total habilidades para la vida & $-0,475^{* *}$ & 0,009 \\
Resiliencia/rasgos frikis & $-0,245^{* *}$ & 0,000 \\
Autoeficacia/rasgos frikis & $-0,238^{* *}$ & 0,000 \\
Autoeficacia/nomofobia & $-0,163^{*}$ & 0,015 \\
Satisfacción vital/síntomas de adicción & $-0,119^{* *}$ & 0,008 \\
Tolerancia a la frustración/síntomas de adicción & $-0,357^{* *}$ & 0,001 \\
Tolerancia a la frustración/uso social & $-0,230^{* *}$ & 0,001 \\
Tolerancia a la frustración/nomofobia & $-0,223^{* *}$ & 0,001 \\
\hline rion
\end{tabular}

** La correlación es significativa al nivel 0,01 (bilateral)

* La correlación es significativa al nivel 0,05 (bilateral)

\section{DISCUSIÓN}

Los resultados del estudio indican un bajo nivel de habilidades para la vida en la muestra del estudio, lo cual es preocupante considerando que sus participantes se encuentran en la edad de adolescencia, siendo de gran vulnerabilidad psicológica y emocional. En este aspecto, un buen nivel de manejo de habilidades para la vida permite contar con herramientas a nivel personal para hacer frente a múltiples dificultades que se presentan en esta edad. 
Los hallazgos del presente estudio van en la misma línea que el de Díaz-Alzate y Mejía-Zapata (2018), realizado, también, en la población de adolescentes en la ciudad de Medellín, que resalta las dificultades en las habilidades para la vida en su muestra de estudio, las cuales podrían estar relacionadas con insuficiencias en los programas psicoeducativos dirigidos a su respectivo fomento.

Por otro lado, los resultados indican que las mujeres adolescentes de la muestra manifestaron mejores habilidades para la vida que los hombres en todas las diez dimensiones constitutivas. Lo anterior podría relacionarse con una mayor madurez psicosocial en mujeres adolescentes, indicada por algunos autores (Rodrigo \& Andreu, 2017). Igualmente, los hallazgos del presente estudio se podrían relacionar con lo planteado por Weichold et al. (2012), quienes indican que las mujeres adolescentes aprovechan más los programas formativos relacionados con las habilidades para la vida y, por ende, cuentan con mayor fortaleza en este aspecto que los hombres.

En la muestra del estudio se identificó un alto nivel de apego a los iguales. Este hecho es consistente con la edad de adolescencia, donde se presenta una alta orientación hacia la relación con los pares, la necesidad de la aprobación social y la dependencia de la opinión social como características distintivas de esta edad evolutiva (Delgado et al., 2011). En esta variable tampoco se identificó la diferencia entre los géneros, aunque algunos estudios indican un mayor grado de apego a los iguales en el género femenino (Delgado et al., 2011).

En cuanto a la resiliencia, autoeficacia y habilidades sociales se obtuvo una baja puntuación y en el autoestima, satisfacción vital y tolerancia a la frustración los niveles fueron medio-bajos, indicando, de este modo, deficiencias en este aspecto en los adolescentes de la muestra. Lo anterior señala una situación preocupante en cuanto al bajo nivel del desarrollo de sus habilidades socioemocionales, lo cual, igualmente, se relaciona con el bajo nivel de habilidades para la vida mostrado por los participantes del estudio. Esta situación relacionada con el bajo nivel del desarrollo de las habilidades blandas en adolescentes contemporáneos, es mencionada por algunos autores que analizan las problemáticas del sistema educativo contemporáneo, resaltando que la excesiva orientación a las habilidades cognitivas y adquisición de conocimientos con miras a los altos estándares de la competencia escolar en el rendimiento de evaluaciones estandarizadas, dejan de lado la importancia del desarrollo de las habilidades socioemocionales en 
los adolescentes contemporáneos (Colom \& Fernández, 2009; Maturana \& Guzmán, 2019).

Por otro lado, los adolescentes de la muestra pertenecientes al género masculino mostraron mayores puntajes en autoestima, autoeficacia, satisfacción vital y tolerancia a la frustración que las mujeres. En este aspecto, los resultados de este estudio van en la misma línea que los hallazgos de los estudios que indican la prevalencia de una mayor autoestima en hombres (Marrero et al., 2003), relacionada, a su vez, con patrones de socialización masculina (Olson \& Shults, 1994; Bleidorn et al., 2016); una mayor tolerancia a la frustración (Chih-Hung et al., 2008) y mayor grado en la satisfacción vital en los hombres adolescentes (Barcelata \& Rivas, 2016).

Es posible que menores puntajes en las variables de autoestima, autoeficacia, satisfacción vital y la tolerancia a la frustración en las adolescentes de la muestra podrían estar relacionados con una mayor vulnerabilidad emocional femenina, sobre todo en la edad de adolescencia, debido a la incidencia de hormonas femeninas en la frecuencia de trastornos afectivos (Van Goozen et al. 1995; Schmidt et al., 1998). Algunos autores afirman que las mujeres, iniciando desde la adolescencia, tienen un mayor nivel de problemas emocionales, mostrando, a lo largo de la vida, mayores trastornos de la afectividad que los hombres (Ortuño, 2014; Dorn et al., 2008; Chaplin et al., 2009; Patton et al., 1996; Huerta, 2003).

En la muestra del presente estudio se identificó una mayor resiliencia en el género femenino, lo cual está acorde con algunos estudios que indican mayores niveles de resiliencia en las mujeres (González-Arratia \& Valdez, 2013). Sin embargo, hay estudios que muestran la presencia de una mayor resiliencia en el género masculino en la edad adolescente (Ramírez-Granizo \& Castro-Sánchez, 2018) o la ausencia de respectivas diferencias (Prado \& Del Águila, 2003).

Lo anterior indica que esta no concordancia en resultados de diferentes estudios podría estar relacionada con distintos contextos socioculturales $\mathrm{u}$ otros factores afines. En caso del presente estudio, un mayor nivel de resiliencia, mostrado por las mujeres, podría relacionarse con el hecho de que ellas en este estudio revelaron, también, mayores habilidades para la vida que los hombres, lo cual indica que cuentan con mayores recursos personológicos para enfrentar las situaciones de adversidad. 
Igualmente, un mayor nivel de habilidades sociales en las mujeres de la muestra del presente estudio está acorde con un mayor puntaje en las habilidades para la vida, siendo estos dos constructos afines entre sí en aspectos de relaciones interpersonales y comunicación efectiva y asertiva.

Al respecto del riesgo de adicción a redes sociales e Internet, los resultados mostraron niveles medios en total de riesgo de adicción, al igual que en las subescalas de síntomas de adicción que valora la presencia de conductas de adicción a sustancias no tóxicas; en el uso social, que muestra el grado de presencia de conductas habituales relacionadas con la "socialización virtual" de los adolescentes, como consultar perfiles, utilizar el chat, comentar fotos, etc.; y nomofobia que indica el grado de presencia de ansiedad en el uso del móvil y el temor de estar "desconectado". Solo en la subescala de rasgos frikis, que refleja la tendencia al uso de juegos virtuales y de rol, unirse a grupos con intereses específicos, buscar encuentros sexuales virtuales, entre otros, se obtuvo un puntaje medio bajo. Lo anterior indica un frecuente empleo de medios sociales e Internet por parte de los adolescentes de la muestra, con un nivel de riesgo medio. Los hallazgos de este estudio se aproximan a los valores obtenidos por Peris et al. (2018) en la población de adolescentes españoles.

En este orden de ideas, algunos autores resaltan altos niveles de presencia de comportamientos adictivos y compulsivos al respecto de medios virtuales en adolescentes, en distintos países, como, por ejemplo, el estudio de Fitria et al. (2018) en la población de Indonesia; el de Rębisz y Sikora (2016), quienes indican que dos tercios de su muestra de adolescentes polacos presentaron un nivel superior al promedio en la adicción al Internet. Durkee et al. (2012) revelaron en un estudio en 11 países europeos que un 3,5\% de los adolescentes encuestados cuenta con uso patológico de Internet y un $13,5 \%$, con consecuencias negativas en su uso. Y el estudio de Mok et al. (2014) indica que $17,9 \%$ de adolescentes coreanos tiene adicción a smartphone. Igualmente, hay estudios que no reportan altos niveles de riesgo de adicción a medios virtuales e internar, como, por ejemplo, los de Ruiz-Palmero et al. (2016) y Marín et al. (2015), en la población española. Estas diferencias en resultados podrían deberse a la incidencia de factores socioeconómicos y culturales, indicando la necesidad de profundizar en esta línea de investigación.

En cuanto a la diferencia de riesgo de adicción a redes sociales e Internet según el género, los resultados del presente estudio confirman los hallazgos del estudio de Peris et al. (2018) en la población de adolescentes españoles, mostrando una diferencia estadísticamente significativa en síntomas de 
adicción, uso social y nomofobia a favor del género femenino, y en rasgos frikis a favor del género masculino.

En esta línea hay estudios que indican que las mujeres tienen una tendencia de estar registradas en múltiples redes sociales (Espinar \& González, 2009), buscando más vínculos sociales, siendo los hombres más orientados a usar juegos virtuales o de rol, al igual que utilizar la virtualidad con propósitos sexuales (Regnerus et al., 2016). Sin embargo, en cuanto a la relación del género con adicción a Internet hay discrepancia entre los estudios, ya que mientras algunos indican que las mujeres adolescentes muestran mayores puntajes debido a su más alta tendencia a publicar y revisar perfiles en línea (Sarabia \& Estévez, 2016); otros afirman la mayor prevalencia de riesgo en hombres (Müller et al., 2016; Durkee et al., 2012), mostrando más altos indicadores en la obsesión por redes sociales en hombres (Araújo, 2016) y una mayor distancia con las redes sociales en las mujeres (Marín et al., 2015).

En cuanto a la diferencia en riesgo de adicción a redes sociales e Internet según el rango de edad, en el presente estudio no se identificó la diferencia estadísticamente significativa entre los grupos de adolescencia temprana y tardía, indicando que el nivel de riesgo no varía con la edad. Esto resultados no concuerdan con lo obtenido en el estudio de Peris et al. (2018), quienes registran mayores puntuaciones en total de riesgo de adicción, síntomas de adicción y nomofobia en adolescentes de 12-14 años en comparación con los de 15-17. En relación a lo anterior, algunos autores consideran que a menor edad hay mayor nivel de riesgo de adicción en general debido a una mayor inmadurez de mecanismo de control de impulsos (Pratarelli \& Browne, 2002; Shek \& Yu, 2016). Sin embargo, considerando tanto los resultados del presente estudio, como la evidencia proporcionada por otras investigaciones, que indican el aumento de adicción a Internet con la edad (Karacic \& Oreskovic, 2017), es necesario ir profundizando en el caso específico de adicción a medios virtuales e Internet y su comportamiento en diferentes edades, por la probable presencia de factores psicosociales y culturales que podrían tener importante incidencia a la par de los determinantes biológicos.

En la comparación del riesgo de adicción a redes sociales e Internet según las variables de tenencia de celular, computador y acceso a Internet se identificó que los adolescentes que cuentan con un celular propio tienen puntajes significativamente mayores en la variable de uso social de redes. Por otro lado, que tenían un computador propio puntuaron significativamente más alto en la variable de rasgos frikis, lo cual indica su mayor disponibilidad para usarlo con fines de juegos virtuales, búsqueda de páginas eróticas u otros 
tipos de entretenimiento. Igualmente, se encontró que los que no cuentan en su lugar de vivienda con servicio de Internet obtuvieron puntajes más altos en síntomas de adicción, rasgos frikis y total de riesgo de adicción. Lo anterior puede estar relacionado con el uso del celular con datos de navegación en Internet que les permiten cierta independencia de lugares como casa o el colegio, donde pueden contar o no con el acceso a Internet, y tener una conexión on-line permanente en todo lugar y tiempo. Estos hallazgos pueden sugerir que no se debe proporcionar a los adolescentes y niños un acceso temprano a medios tecnológicos como celular o computador con el fin de prevenir el riesgo de adicción a redes sociales e Internet. Sin embargo, es importante tener en cuenta que los medios tecnológicos son solo los medios, por lo cual es necesario concientizar acerca de su uso responsable y controlado, sobre todo en la sociedad contemporánea donde se han vuelto completamente imprescindibles para el funcionamiento social.

En cuanto a la diferencia en el riesgo de adicción a redes sociales e Internet según el tipo de familia de los participantes, se identificó la diferencia estadísticamente significativa en la variable de uso social, indicando el mayor promedio en el grupo que vive con abuelos, seguido por el grupo que vive con la madre y con el menor promedio en el grupo que vive con ambos padres. Lo anterior puede estar relacionado con la disponibilidad de tiempo libre de los adolescentes y el grado de supervisión de los cuidadores en distintos tipos de familia sobre sus actividades.

Finalmente, los datos del presente estudio indican la correlación mediana y negativa entre las variables de total riesgo de adicción a redes sociales e Internet y el total de las habilidades para la vida, señalando que a menor desarrollo de las habilidades para la vida existe mayor riesgo de adicción a redes sociales e Internet. Considerando que las habilidades para la vida son herramientas para afrontar distintas situaciones de la vida cotidiana y solucionar problemas, se podría pensar que los pasatiempos de revisar novedades y chismes en redes sociales y entretenimiento en Internet como juegos en línea y de roles, encuentros sexuales, entre otros, podría emplearse como una forma de evadir estas dificultades y problemas de la vida real, en ausencia de recursos personológicos relacionados con las habilidades para la vida, para su respectivo afrontamiento funcional.

Sin embargo, el constructo de habilidades para la vida es bastante complejo, siendo incluidas diez dimensiones en el modelo teórico utilizado en el presente estudio (OMS, 1992). Así que es necesario mirar de forma más 
detallada cómo se relaciona cada una de las variables de riesgo de adicción con distintas dimensiones constitutivas de las habilidades para la vida.

Por ejemplo, la correlación negativa obtenida entre la variable de rasgos frikis y las variables de empatía y del manejo de emociones, indica que los adolescentes de la muestra que cuentan con mayores deficiencias en estas dos dimensiones tienen mayor tendencia a utilizar el Internet en pasatiempos de juegos virtuales, buscar información sobre sexualidad, entrar en páginas eróticas y tener encuentros sexuales.

Por otro lado, la variable de uso de redes sociales se correlacionó de forma negativa con la dimensión de manejo de tensiones y estrés, lo cual sugiere que el uso compulsivo de redes sociales podría ser una forma de distracción para evitar las situaciones estresantes de la vida real.

En este aspecto, Challco et al. (2016) en un estudio en la población de adolescentes encontraron una relación significativa inversa entre el riesgo de adicción a redes sociales, la autoestima y el autocontrol, concluyendo que el aislamiento y poco dominio sobre su propia conducta posibilitan el aumento de adicción a redes sociales.

Por otro lado, los hallazgos del presente estudio arrojaron correlaciones positivas entre la variable de uso de redes sociales y las dimensiones de las habilidades para la vida como: conocimiento de sí mismo, empatía, comunicación efectiva y asertiva, relaciones interpersonales, toma de decisiones, solución de problemas y conflictos, pensamiento creativo, pensamiento crítico, y manejo de sentimientos y emociones. Las correlaciones anteriores, aunque siendo bajas, fueron significativas, lo cual amerita considerarlas dentro de los resultados del estudio.

Con base en los aspectos valorados en esta variable de uso social de redes sociales se puede ver que esta no solo implica consultar perfiles y/o subir fotos, o entretenerse con los chismes sociales, sino también, hacer nuevos amigos, utilizar el chat para establecer las relaciones interpersonales, encontrar e interactuar con amigos antiguos, buscar parejas posibles, entre otras, actividades que, aunque se realizan en un espacio virtual, hacen parte de relaciones interpersonales. Los hallazgos del presente estudio indican que el uso de redes sociales virtuales, al igual que el manejo de las situaciones de interacción interpersonal presencial, requieren tanto del manejo de las habilidades socioemocionales (conocimiento de sí mismo, empatía, comunicación efectiva y asertiva, relaciones interpersonales, manejo de 
sentimientos y emociones), como cognitivas (pensamiento crítico y creativo, toma de decisiones, solución de problemas y conflictos). Los adolescentes de la muestra que cuentan con un mejor manejo de estas dimensiones de las habilidades para la vida también tienen una tendencia a mayor uso social de redes sociales. En este aspecto se podría considerar, tal como plantean algunos autores, que no siempre el uso de los medios virtuales debe considerarse como algo malo (Pérez, 2016). El hecho de que este uso puede convertirse en problemático generando síntomas de adicción, al igual como en otro tipo de adicciones comportamentales, está relacionado con otros factores de índole psicosocial o biológica que generan vulnerabilidad psicológica, sobre todo en la edad adolescente. Además, es necesario tener en cuenta que el uso de medios virtuales está en aumento progresivo y será cada vez mayor en los próximos años, lo cual indica la necesidad de llevar a cabo los estudios orientados a identificar qué tipo de deficiencias en los recursos personológicos podrían estar relacionados con el aumento del riesgo de adicción, al igual que cuáles fortalezas podrían ayudar a un manejo adecuado de este recurso virtual de comunicación con los demás.

En cuanto a la correlación entre variables de riesgo de adicción y otras variables socioemocionales, se identificaron las correlaciones negativas entre la variable rasgos frikis y resiliencia y autoeficacia, indicando que el mayor uso de medios virtuales relacionado con entretenimientos de juegos en línea y sitios eróticos tiene referencia con dificultades a nivel de autoeficacia y de las habilidades resilientes.

La variable de nomofobia o el temor de estar desconectado, mostró una correlación negativa con autoeficacia y tolerancia a la frustración, indicando que bajos niveles de autoeficacia y tolerancia a la frustración están relacionados con mayores niveles de ansiedad y la necesidad de estar conectado on-line de forma permanente.

Y la variable de síntomas de adicción obtuvo una correlación negativa con la satisfacción vital y tolerancia a la frustración, lo cual sugiere que bajos niveles en satisfacción vital y baja tolerancia a la frustración, podrían estar relacionados con mayor uso de redes sociales virtuales e Internet de forma problemática. En este aspecto, los resultados del presente estudio están acorde con lo sugerido por Chih-Hung et al. (2008), quienes indican que bajos niveles de tolerancia a la frustración están relacionados con la adicción a Internet, sobre todo en el sexo masculino. 


\section{CONCLUSIÓN}

A nivel general, los resultados del presente estudio, además de indicar un nivel medio de riesgo de adicción a redes sociales e Internet en la muestra del estudio, sugieren que las deficiencias en las habilidades blandas de los adolescentes podrían estar relacionadas con el aumento de este riesgo. Lo anterior va en línea con otros estudios cuyos hallazgos indican que los que no cuentan con un adecuado desarrollo de sus habilidades socioemocionales tienen tendencia a evidenciar los síntomas de adicción a las redes sociales e Internet (Domínguez \& Ybañez, 2016; Romo, 2018; Carmona et al., 2020; Estrada et al., 2021).

En este aspecto es necesario considerar que el uso de medios virtuales en la vida diaria es cada vez mayor, sobre todo en adolescentes, y, por ende, requiere de un buen manejo de las habilidades socioemocionales, permitiendo lograr un buen uso de esta herramienta, sin desarrollar riesgo de adicción (Terán Prieto, 2019). Los resultados de este estudio apoyan la importancia del fomento, desde los ambientes educativos, de las habilidades socioemocionales y las habilidades para la vida como un posible factor protector para el manejo responsable y cuidadoso del mundo virtual y prevención del riesgo de adicción, siendo este un tema resaltado por muchos autores, quienes indican que la educación no solo debe proporcionar a los estudiantes el conocimiento sobre el manejo de medios tecnológicos, sino también asegurar que adquieran el desarrollo necesario y suficiente de habilidades blandas para poder prevenir el riesgo de adicción en el uso de estos medios (Ortiz et al., 2020; Sánchez et al., 2018).

$\mathrm{Y}$, por último, es necesario indicar que el presente estudio tuvo limitaciones en cuanto al tamaño de la muestra, sugiriendo la necesidad de estudiar este fenómeno en muestras más amplias y otros contextos culturales y socioeconómicos.

Conflicto de interés: Los autores del presente escrito declaran que no existe ningún conflicto de intereses de tipo laboral contractual o personal que pudieran ocasionar un sesgo no intencionado en el proceso investigativo realizado.

Agradecimiento: Se expresan agradecimientos al colegio público La Paz, del municipio de Envigado, y expresamente al psicólogo Diego Torres, por proporcionar las condiciones necesarias para la realización del estudio.

Financiamiento: La financiación del presente estudio se realizó de forma independiente por cuenta de los investigadores. 


\section{REFERENCIAS}

Alfaro, J., Guzmán, J., Sirlopú, D., García, C., Reyes, F., \& Gaudlitz, L. (2016). Propiedades psicométricas de la Escala de Satisfacción con la Vida en los Estudiantes (SLSS) de Huebner en niños y niñas de 10 a 12 años de Chile. Anales de Psicología, 32(2), 383-392. https://dx.doi.org/10.6018/analesps.32.2.217441

American Psychiatric Association (APA), (2013). Diagnostic and statistical manual of mental disorders: DSM-5. 5th ed. American Psychiatric Association.

Ascher, M., \& Levounis, P. (2015). The behavioral addictions. American Psychiatric Association Publishing.

Araujo, E. D. (2016). Indicadores de adicción a las redes sociales en universitarios de Lima. Revista Digital de Investigación en Docencia Universitaria, 10(2), 48-58. http://dx.doi.org/10.19083/ridu.10.494

Arias, O., Gallego, V., Rodríguez, M. \& Del Pozo, M. (2012). Adicción a las nuevas tecnologías. Psicología de las Adicciones, 1, 2-6, https://www.unioviedo.es/gca/ uploads/pdf/Psicologia\%20de\%20las\%20Adicciones,\%20vol.1,\%202012.pdf

Armsden, G., \& Greenberg, M. (1987). The inventory of parent and peer attachment: Individual differences and their relationship to psychological well-being in adolescence. Journal of Youth and Adolescence, 16, 427-454. https://doi.org/10.1007/ BF02202939

Basteiro, J., Robles-Fernández, A., Juarros-Basterretxea, J. \& Pedrosa, I. (2013). Adicción a las redes sociales: creación y validación de un instrumento de medida. Revista de Investigación y Divulgación en Psicología y Logopedia, 3(1), 2-8. https://www.ridpsiclo.ull.es

Baessler, J. \& Schwarzer, R. (1996). Evaluación de la autoeficacia: adaptación española de la escala de autoeficacia general. Ansiedad y estrés, 2(1), 1-8. https://dialnet. unirioja.es/servlet/articulo?codigo $=186652$

Barcelata, B. \& Rivas, D. (2016). Bienestar psicológico y satisfacción vital en adolescentes mexicanos tempranos y medios. Revista Costarricense de Psicología, 35(2), 121-139. http://www.rcps-cr.org/openjournal/index.php/RCPs/article/view/84

Bar-On, R. \& Parker, J.D.A. (2000). The Bar-On Emotional Quotient Inventary: Youth Version (EQ:YV): Technical Manual. Toronto, Canada Multi-health Systems

Becoña, E. \& Oblitas, L. (2003). Psicología de la salud y adicciones: perspectiva terapéutica. Revista de Psicología, 21(1), 71-106. http://revistas.pucp.edu.pe/ index.php/psicologia/article/view/3719

Becoña, E. (2016). La adicción "no" es una enfermedad cerebral. Papeles del Psicólogo, 37(2), 118-125. http://papelesdelpsicologo.es/pdf/2696.pdf

Becoña, E. (2018). Adicciones comportamentales: separando el grano de la paja. INFONOVA, Revista profesional y académica sobre adicciones, 34, 11-21. https:// dianova.es/wp-content/uploads/2018/06/Infonova_34_web.pdf

Bleidorn, W., Denissen, J., Gebauer, J., Arslan, R., Rentfrow, P., Potter, J., \& Gosling, S. (2016). Age and Gender Differences in Self-Esteem-A Cross-Cultural Window. Journal of Personality and Social Psychology, 111(3), 396-410. http://dx.doi. org/10.1037/pspp0000078

Carbonell, X. \& Oberst, U. (2015). Las redes sociales en línea no son adictivas. Aloma, Revista de Psicologia, Ciències de l'Educació i de l'Esport, 33(2), 13-19. https:// www.raco.cat/index.php/Aloma/article/viewFile/301478/391079 
Caro, M. (2017). Adicciones tecnológicas: ¿Enfermedad o conducta adaptativa?. MediSur, 15(2), 251-260. http://scielo.sld.cu/scielo.php?script=sci_arttext\&pi$d=\$ 1727-897 X 2017000200014 \& \operatorname{lng}=$ es\&tlng=es

Carmona, P., Mesa, J., Patiño, J. \& Klimenko, O. (2020). Riesgo de adicción a redes sociales e Internet y su relación con las habilidades sociales y el bienestar psicológico en estudiantes de 9 a 11 grado de instituciones educativas públicas y privadas del municipio La Ceja. [Trabajo de grado, Especialización en Adicciones, Universidad Católica Luis Amigó]. http://repository.ucatolicaluisamigo.edu. co:8080/jspui/handle/ucatolicaamigo/384

Cia, A. (2013). Las adicciones no relacionadas a sustancias (DSM-5, APA, 2013): un primer paso hacia la inclusión de las Adicciones Conductuales en las clasificaciones categoriales vigentes. Rev Neuropsiquiatr 76(4), 210-217. www.upch.edu. pe

Challco, K., Rodríguez, S. \& Jaimes, J. (2016). Riesgo de adicción a redes sociales, autoestima y autocontrol en estudiantes de secundaria. Revista Científica de Ciencias de la Salud, 9(1), 9-15. https://doi.org/10.17162/rccs.v9i1.542

Chaplin, T., Gillham, J., \& Seligman, M. (2009). Gender, Anxiety, and Depresive Symptoms: A Longitudinal Study of Early Adolescents. Journal of Early Adolescence, 29(2), 307-327. https://doi.org/10.1177/0272431608320125

Chóliz, M. (2010). Mobile phone addiction: a point of issue. Addiction, 105(2), 373-374. https://doi.org/10.1111/j.1360-0443.2009.02854.x

Chóliz, M. \& Villanueva, V. (2011). Evaluación de la adicción al móvil en la adolescencia. Revista Española de Drogodependencias, 36(2), 165-184. https://www. aesed.com/descargas/revistas/v36n2_3.pdf

Chih-Hung, K., Ju-Yu, Y., Cheng-Fang, Y., Chung-Sheng, Ch., \& Shing-Yaw, W. (2008). The association between Internet addiction and belief of frustration intolerance: the gender difference. Cyberpsychol Behavior, 11(3), 273-278. https://doi. org/10.1089/cpb.2007.0095

Colom, J. \& Fernández, M. (2009). Adolescencia y desarrollo emocional en la sociedad actual. International Journal of Developmental and Educational Psychology, 1(1), 235-242. https://www.redalyc.org/pdf/3498/349832320025.pdf

Davis, R. (2001). A cognitive-behavioral model of pathological Internet use. Computers in Human Behavior, 17(2), 187-195. https://doi.org/10.1016/S07475632(00)00041-8

Delgado, I., Oliva, A. \& Sánchez-Queija, I. (2011). Apego a los iguales durante la adolescencia y la adultez emergente. Anales de Psicología, 27(1), 155-163. https://www.redalyc.org/articulo.oa?id=167/16717018018

Díaz, L., Rosero, R., Melo, M. \& Aponte, D. (2013). Habilidades para la vida: análisis de las propiedades psicométricas de un test creado para su medición. Revista Colombiana de Ciencias Sociales, 4(2), 181-200. https://dialnet.unirioja.es/ servlet/articulo?codigo $=5123783$

Díaz-Alzate, M. \& Mejía-Zapata, S. (2018). La mirada de los adolescentes al modelo de habilidades para la vida. Revista Latinoamericana de Ciencias Sociales, Niñez y Juventud, 16(2), 709-718. https://doi.org/10.11600/1692715x.16205

Domínguez J. \& Ybañez, J. (2016). Adicción a las redes sociales y habilidades sociales en estudiantes de una institución educativa privada. Propósitos y Representaciones, 4(2), 181-230. http://dx.doi.org/10.20511/pyr2016.v4n2.122

Dorn, L., Negriff, S., Huang, B., Pabst, S., Hillman, J. Braverman, P., \& Susman, E. (2009). Menstrual Symptoms in Adolescent Girls: Association with Smoking, 
Depressive Symptoms and Anxiety. J Adolesc Health, 44(3), 237-243. https://doi. org/10.1016/j.jadohealth.2008.07.018

Durkee, T., Kaess, M., Carli, V., Parzer, P., Wasserman, C., Floderus, B., Apter, A., Balazs, J., Barzilay, S., Bobes, J., Brunner, R., Corcoran, P., Cosman, D., Cotter, P., Despalins, R., Graber, N., Guillemin, F., Haring, C., Kahn, J.P., Mandelli, L., Marusic, D., Mészáros, G., Musa, G. J., Postuvan, V., Resch, F., Saiz, P. A., Sisask, M., Varnik, A., Sarchiapone, M., Hoven, Ch. W. \& Wasserman, D. (2012). Prevalence of pathological Internet use among adolescents in Europe: demographic and social factors. Addiction, 107(12), 2210-2222. https://doi.org/10.1111/j.13600443.2012.03946.x

Echeburúa, E. \& De Corral, P. (2010). Adicción a las nuevas tecnologías y a las redes sociales en jóvenes: un nuevo reto. Adicciones, [S.I.], 22(2), 91-96, http://www. adicciones.es/index.php/adicciones/article/view/196/186

Escobar, Y. \& Zambrano, D. (2015). Propiedades psicométricas de la escala de autoeficacia general (Baessler \& Schwarzer, (1996) en una muestra de adolescentes de San Juan de Pasto. [Tesis de grado, Psicología. Universidad de Nariño]. http:// biblioteca.udenar.edu.co:8085/atenea/biblioteca/90702.pdf

Escurra, M. \& Salas, E. (2014). Construcción y validación del cuestionario de Adicción a Redes Sociales (ARS). Liberabit. Revista de Psicología, 20(1), 73-91. http://www. redalyc.org/articulo.oa?id=68631260007

Espinar, E. \& González, M. J. (2009). Jóvenes en las redes sociales virtuales: un análisis exploratorio de las diferencias de género. Feminismo/s, 14, 87-106. https://doi. org/10.14198/fem.2009.14.06

Estrada, E., Zuloaga, M., Gallegos, N. \& Mamani, H. (2021). Adicción a Internet y habilidades sociales en adolescentes peruanos de educación secundaria. Archivos Venezolanos de Farmacología y Terapéutica, 40(1), 74-80. https://www.revistaavft.com/images/revistas/2021/avft_1_2021/14_adiccion_internet_habilidades.pdf

Fernández, N. (2013). Trastornos de conducta y redes sociales en Internet. Salud Mental, 36, 521-527. http://www.scielo.org.mx/pdf/sm/v36n6/v36n6a10.pdf

Ferre, F., Sevilla, J. \& Basurte, I. (2016). Protocolos de intervención. Adicciones comportamentales y patología dual. EdikaMed, S.L. https://patologiadual.es/ wp-content/uploads/2019/02/8-pdual-adicciones.pdf

Fitria, L., Ifdil, I., Erwinda, L., Ardi, Z., Afdal, A., Sari, A., Fadli, R., Rangka, I., Suranata, K., Bariyyah, K., Zola, N., \& Churnia, E. (2018). Exploring Internet addiction on adolescents. Journal of Physics: Conf. Series 1114, 012076. https://doi. org/10.1088/1742-6596/1114/1/012076

Foster, A., Shorter, G., \& Griffitsh, M. (2015). Muscle dysmorphia: Could it be classified as an addiction to body image? Journal of Behavioral Addiction, 4, 1-5. https:// doi.org/10.1556/JBA.3.2014.001

Flores, N., Jenaro, C., González, F., Martín, E. \& Poy, R. (2013). Adicción al móvil en alumnos de secundaria: efectos en la convivencia. European Journal of Investigation in Health, Psychology and Education, 3(3), 215-225. https://doi. org/10.30552/ejihpe.v3i3.47

Fuertes, J. \& Armas, L. (2018). Adolescentes adictos a redes sociales y tecnología. Horizontes de Enfermería, [S.I.], 7, 155-166. http://revistasdigitales.upec.edu.ec/ index.php/enfermeria/article/view/163

González, N. (2016). Resiliencia y personalidad en niños y adolescentes. Ediciones y Gráficos Eón, S.A. de C.V. http://ri.uaemex.mx/handle/20.500.11799/64951 
Gonzales, R. (2015). Las adicciones comportamentales: una tormenta al acecho. Revista del Hospital Psiquiátrico de La Habana, 12(S1). https://www.medigraphic. com/cgi-bin/new/resumen.cgi?IDARTICULO=64576

González-Arratia, N. \& Valdez, J. (2013). Resiliencia: Diferencias por edad en hombres y mujeres mexicanos. Acta de Investigación Psicológica, 3(1), 941-955. https:// doi.org/10.1016/S2007-4719(13)70944-X

González-López, A. \& Naranjo-Fundora, W. (2013). ¿Cuáles son los factores de riesgo adictivo en la adolescencia? Apuntes para maestros de la enseñanza media. EduSol, 13(43), 11-21. http://www.redalyc.org/pdf/4757/475748682002.pdf

Grant, J., Odlaug, B., \& Chamberlain, S. (2016). Why can't I stop? John Hopkins Press.

Griffiths, M., Kuss, D., \& Demetrovics, Z. (2014). Social networking addiction: An overview of preliminary findigns. Chapter 6, In K. P. Rosenberg y L. C. Feder (Eds.), Behavioral addictions. Criteria, evidence, and treatment. (pp. 119-141). Academic Press. https://doi.org/10.1016/B978-0-12-407724-9.00006-9

Han, D., Lee, Y., Yang, K., Kim, E., Lyoo, I., \& Renshaw, P. (2007). Dopamine genes and reward dependence in adolescents with excessive Internet video game play. J Addict Med, 1(3), 133-138. https://doi.org/10.1097/ADM.0b013e31811f465f

Huebner, E. S. (1991). Further validation of the Students Life Satisfaction Scale: The independence of satisfaction and affect rating. Journal of Psychoeducational Assessment, 9, 363-368. https://doi.org/10.1177/073428299100900408

Huerta, M. (2003). El estado de ánimo de la mujer durante su ciclo reproductivo. Salud Mental, 23(3), 52-60. https://www.medigraphic.com/pdfs/salmen/ sam-2000/sam003g.pdf

Hormes, J., Kearns, B. \& Timko, C. (2014). Craving Facebook? Behavioral addiction to online social networking and its association with emotion regulation deficits. Addiction, 109(12), 2079-2088. https://doi.org/10.1111/add.12713

Huang, M., \& Alessi, N. (1997). Internet addiction, Internet psychotherapy. Am J Psychiatry, 153(6), 890. https://ajp.psychiatryonline.org/doi/pdfplus/10.1176/ ajp.154.6.890

Karacic, S., \& Oreskovic, S. (2017). Internet Addiction Through the Phase of Adolescence: A Questionnaire Study. JMIR Mental Health, 4(2), e11. https://doi. org/10.2196/mental.5537

Kardefelt-Winther, D., Heeren, A., Schimmenti, A., Van Rooij, A., Maurage, P., Carras, M., Edman, J., Blaszczynski, A., Khazaal, Y., \& Billieux, J. (2017). How can we conceptualize behavioural addiction without pathologizing common behaviours? Addiction, 112(10), 1709-1715. https://doi.org/10.1111/add.13763

Kiefer, F., Fauth-Bühler, M., Heinz, A., \& Mann, K. (2017). Neurobiology of behavioral addictions. Nervenarzt, 84(5), 557-562. https://doi.org/10.1007/s00115-0123719-y

Lopez-Fernandez, O., Honrubia-Serrano, L., Freixa-Blanxart, M., \& Gibson, W. (2014). Prevalence of problematic mobile phone use in British adolescents. Cyberpsychology, Behavour and Social Networking, 17(2), 91-98. https://doi.org/10.1089/ cyber.2012.0260

Marín, V., Sampedro, B. \& Muñoz, J. (2015). ¿Son adictos a las redes sociales los estudiantes universitarios? Revista Complutense de Educación, 26, 233-251, http://dx.doi.org/10.5209/rev_RCED.2015.v26.46659 
Marrero, R., Matud, M., Carballeira, M. \& Ibáñez, I. (2003). Diferencias de autoestima en función del género. Análisis y modificación de conducta, 29(123), 51-78. https://dialnet.unirioja.es/servlet/articulo?codigo=649436

Martínez, G. \& Alfaro, A. (2019). Validación de la escala de autoestima de Rosenberg en estudiantes paceños. Fides et Ratio - Revista de Difusión cultural y científica de la Universidad La Salle en Bolivia, 17(17), 83-100. http://www.scielo.org.bo/ scielo.php?script=sci_arttext\&pid=S2071-081X2019000100006\&lng=es\&tlng=es

Magura, S., \& Rosenblum, A. (2000). Modulating effect of alcohol use on cocaine use. Addictive Behavior, 25(1), 117-22. https://doi.org/10.1016/s03064603(98)00128-2

Maturana, G. \& Guzmán, F. (2019). Las competencias blandas como complemento de las competencias duras en la formación escolar. Revista EURITMIA - Investigación, Ciencia y Pedagogía, 1, 1-84, http://cliic.org/pdf/Revista\%20Euritmia,\%20 Vol.\%201\%20N\%C2\%B01.pdf

Méndez-Díaz, M., Romero, B., Cortés, J., Ruíz-Contreras, A. \& Prospéro-García, O. (2017). Neurobiología de las adicciones. Revista de la Facultad de Medicina de la UNAM, 60(1), 6-16. https://www.medigraphic.com/pdfs/facmed/un-2017/ un171b.pdf

Mendoza, Y. \& Vargas, K. (2017). Factores psicosociales asociados al consumo y adicción a sustancias psicoactivas. Revista Electrónica de Psicología, Iztacala, 20(1), 139-167. http://www.iztacala.unam.mx/carreras/psicologia/psiclin/ vol20num1/Vol20No1Art9.pdf

Mok, J., Choi, S., Kim, D., Choi, J., Lee, J., Ahn, H., Choi, E., \& Song, W. (2014). Latent class analysis on Internet and smartphone addiction in college students. Neuropsychiatr Dis Treat, 10, 817-828. https://doi.org/10.2147/NDT.S5929 3

Müller, K., Dreier, M., Beutel, M., Duven, E., Giralt, S., \& Wölfing, K. (2016). A hidden type of Internet addiction? Intense and addictive use of social networking sites in adolescents. Computers in Human Behavior, 55, 172- 177. https://doi. org/10.1016/j.chb.2015.09.007

Observatorio Español de las Drogas y las Adicciones (2019). Adicciones comportamentales, juego y uso compulsivo de Internet en las encuestas de drogas y adicciones en España EDADES y ESTUDES. Delegación del Gobierno para el Plan Nacional sobre Drogas, España. http://www.pnsd.mscbs.gob.es/profesionales/sistemasInformacion/sistemalnformacion/pdf/2019_Informe_adicciones_comportamentales.pdf

Oliva, A., Antolín, L., Pertegal, M., Ríos, M., Parra, A., Gómez, A. \& Reina, M. (2011). Instrumentos para la evaluación de la salud mental y el desarrollo positivo adolescente y los activos que lo promueven. Junta de Andalucía. Consejería de Salud. https://idus.us.es/handle/11441/32153;jsessionid=DFF81492326C51A942B93D653B8D7628?

Olson, D., \& Shults, K. (1994). Gender differences in the dimensionality of social support. Journal of Applied Social Psychology, 24(14), 1221-1232, https://doi. org/10.1111/j.1559-1816.1994.tb00555.x

Ovejero, A. (2000). La adicción como búsqueda de identidad: una base teórica psicosocial para una intervención eficaz. Intervención Psicosocial, 9(2), 199-215. http://www.copmadrid.org/webcopm/publicaciones/social/63247.pdf

Organización Mundial de la Salud (OMS, 1992). Clasificación Estadística Internacional de Enfermedades y Problemas Relacionados con la Salud (CIE-10). http://ais. paho.org/classifications/Chapters/pdf/Volume2.pdf 
Organización Mundial delaSalud(OMS, 1999). Guidelines: LifeSkills Education Curricula for Schools. Ginebra, Suiza. https://apps.who.int/iris/handle/10665/63552

Ortiz, E., Hincapié, D. \& Paredes, D. (2020). Educar para la vida: el desarrollo de las habilidades socioemocionales y el rol de los docentes. Banco Interamericano de Desarrollo. https://publications.iadb.org/publications/spanish/ document/Educar-para-la-vida-El-desarrollo-de-las-habilidades-socioemocionales-y-el-rol-de-los-docentes.pdf

Ortuño, J. (2014). Adolescencia. Evaluación del ajuste emocional y comportamental en el contexto escolar. [Tesis Doctoral, Universidad de La Rioja]. https://dialnet. unirioja.es/servlet/oaites?codigo $=42271$

Patton, G., Hibbert, M., Carlin, J., Shao, Q., Rosier, M., Caust, J., \& Bowes, G. (1996). Menarche and the onset of depression and anxiety in Victoria, Australia.J Epidemiol Community Health, 50(6), 661-666. https://doi.org/10.1136/jech.50.6.661

Pedrero, E. (2010). Detección de adicciones comportamentales en adictos a sustancias en tratamiento. Trastornos adictivos, 12(1), 13-18. https://doi.org/10.1016/ S1575-0973(10)70005-2

Pérez del Río, F. \& Martín, I. (2007). Nuevas adicciones: ¿adicciones nuevas? Intermedio.

Pérez, L. (2016). Metodología para establecer la percepción de beneficios o de desventajas académicas del uso de redes sociales en educación secundaria. [Maestría tesis, Universidad Nacional de Colombia - Sede Bogotá]. http://bdigital.unal.edu. $\mathrm{co} / 56042 /$

Peris, M., Maganto, C. \& Garaigordobil, M. (2018). Escala de Riesgo de Adicción-Adolescente a las Redes Sociales e Internet: fiabilidad y validez (ERA-RSI). Revista de Psicología Clínica con Niños y Adolescentes, 5(2), 30-36. https://doi.org/10.21134/ rpcna.2018.05.2.4

Pert, C., Ruff, M., Weber, R., \& Herkenham, M. (1985). Neuropeptides and their receptors: a psychosomatic network. The Journal of Immunology, 135(2), 820s-826s. https://doi.org/0022-1767/85/1352-820s\$02.00

Pert, C. (1986). La sabiduría de los receptores: neuropéptidos, emociones y cuerpomente. Advances Institute for the Advancement of Health, 3(3), 8-20. https:// seryactuar.files.wordpress.com/2012/12/la-sabidurc3ada-de-las-emociones-neuropc3a9ptidos-emociones-cuerpo-mente-candace-pert-1986-pdf.pdf

Petry, N. (Ed.) (2016a). Behavioral addictions. DSM-5 and beyond. Oxford University Press.

Petry, N. (2016b). Conclusions and future directions to advance the scince and clinical care of behavioral addictions. In N. M. Petry (Ed.), Behavioral addictions. DSM-5 and beyond (pp. 221-230). Oxford University Press.

Pons, X., (2008). Modelos interpretativos del consumo de drogas. Polis - Investigación y Análisis Sociopolítico y Psicosocial, 4(2), 157-186. http://www.scielo.org.mx/ pdf/polis/v4n2/v4n2a6.pdf

Prado, R. \& Del Águila, M., (2003). Diferencia en la resiliencia según género y nivel socioeconómico en adolescentes. Revista de la Facultad de Psicología, 6, 179-196. https://dialnet.unirioja.es/servlet/articulo?codigo=2879576

Pratarelli, M., \& Browne, B. (2002). Confirmatory factor analysis of Internet use and addiction. CyberPsychology and Behavior, 5, 53-64. https://doi. org/10.1089/109493102753685881 
Ramírez-Granizo, I. \& Castro-Sánchez, M. (2018). Análisis de los niveles de resiliencia en función del género y factores del ámbito educativo en escolares. ESHPA Education, Sport, Health and Physical Activity, 2(1), 50-61. http://hdl.handle. net/10481/49838

Rębisz, S., \& Sikora, I. (2016). Internet Addiction in Adolescents. Practice and Theory in Systems of Education, 11(3), 194-204. https://doi.org/10.1515/ptse-2016-0019

Regnerus, M., Gordon, D., \& Price, J. (2016). Documenting pornography use in America: a comparative analysis of methodological approaches. Journal of Sex Research, 53, 873-881. https://doi.org/10.1080/00224499.2015.1096886

Riva-Posse, A. (2016). Trastornos adictivos. Inmanencia, 5(1), 51-57. https://ri.conicet.gov.ar/admin/bitstream/11336/70881/2/CONICET_Digital_Nro.9f08835233da-472a-a8bd-f224d3ff810d_A.pdf

Rodrigo, J. \& Andreu, J. (2017). Evaluación psicológica de la madurez psicosocial en adolescentes. Psicopatología Clínica, Legal y Forense, 17(1), 14-31. https:// dialnet.unirioja.es/servlet/articulo?codigo $=6674241$

Romo, D. (2018). Adicción a Internet y habilidades sociales en estudiantes de secundaria de la Institución Educativa Internacional Elim de Villa El Salvador - 2017. [Trabajo de grado de Máster. Universidad Cesar Vallejo, Perú] https://repositorio. ucv.edu.pe/bitstream/handle/20.500.12692/14122/Romo_SDS.pdf?sequence $=1 \&$ is Allowed $=y$

Rosenberg, M. (1965). Society and Adolescent self-image. University Press.

Ruiz-Palmero, J., Sánchez-Rodríguez, J. \& Trujillo-Torres, J. (2016). Utilización de Internet y dependencia a teléfonos móviles en adolescentes. Revista Latinoamericana de Ciencias Sociales, Niñez y Juventud, 14(2), 1357-1369. https://dx.doi. org/10.11600/1692715x.14232080715

Sánchez-Carbonell, X., Beranuy, M., Castellana, M., Chamarro, A. \& Oberst, U. (2008). La adicción a Internet y al móvil: ¿moda o trastorno? Adicciones, 20(2), 149-159. https://www.redalyc.org/pdf/2891/289122057007.pdf

Sánchez Peña, J. \& Rodríguez Solano, J. (2006). Juego patológico asociado al alcoholismo: un estudio de comorbilidad. An Psiquiatr, 22(2), 72-76. https://medes. com/publication/23067

Sánchez, L., Benito, R., Serrano, A., Benavent, R. \& Bueno, F. (2018). Programa de prevención del uso problemático de Internet y redes sociales. "Clickeando". Unidad de Prevención Comunitaria en Conductas Adictivas (UPCCA-València). http:// www.codajic.org/sites/www.codajic.org/files/Programa-prevencion-uso-problematico-internet-redes-sociales-Clickeando-Web(1).pdf

Sarabia, I., \& Estévez, A. (2016). Sexualized behaviors on Facebook. Computers in Human Behavior, 61, 219-226. https://doi.org/10.1016/j.chb2016.03.0370747-5

Schmidt, P., Nieman, L., Danaceau, M., Adams, L. \& Rubinow, D. (1998). Differential behavioral effects of gonadal steroids in women with and in those without premenstrual syndrome. New Eng J Clinic Med, 338, 209-216. https://doi. org/10.1056/NEJM199801223380401

Shapira, N., Goldsmith, T., \& Keck, P. (1998). Psychiatric evaluation of individuals with problematic use of the Internet. 151st Annual Meeting of the American Psychiatric Association, Toronto. https://www.newswise.com/articles/151st-annualapa-meeting-in-toronto

Shapira, N., Goldsmith, T., Keck, P., Khosla, U., \& McElroy, S. (2000). Psychiatric features of individuals with problematic Internet use. Journal of Affective Disorders, 57(1-3), 267-272. https://doi.org/10.1016/S0165-0327(99)00107-X 
Shek, D., \& Yu, L. (2016). Adolescent Internet addiction in Hong Kong: prevalence, change, and correlates. Journal of Pediatric and Adolescent Gynecology, 29(1), 22-30. https://doi.org/10.1016/j.chb.2016.10.029

Sussman, S. (2017). Substance and behavioral addictions: Concepts, causes, and cures. Cambridge University Press.

Terán Prieto, A. (2019). Ciberadicciones. Adicción a las nuevas tecnologías (NTIC). En AEPap (ed.), Congreso de Actualización en Pediatría 2019. Lúa Ediciones 3.0. pp.131-141. https://www.aepap.org/sites/default/files/pags._131-142_ciberadicciones.pdf

Van Goozen, S., Cohen-Kettenis, P., Gooren, L., Frijda, N., Van de Poll, N. (1995). Gender differences in behavior: Activating effects of cross-sex hormones. Psychoneuroendocrinology, 20, 343-363. https://doi.org/10.1016/0306-4530(94)00076-X

Weinstein, A., Curtiss Feder, L., Rosenberg, K. P., \& Dannon, P. (2014). Internet addiction disorder: Overview and controversies. In K. P. Rosenberg \& L. Curtiss Feder (Eds.), Behavioral addictions: Criteria, evidence, and treatment (pp. 99-117). Elsevier Academic Press. https://doi.org/10.1016/B978-0-12-407724-9.00005-7

Weichold, K., Silbereisen, R., \& Brambosch, A. (2012). Do Girls Profit More? Gender-Specific Effectiveness of a Life Skills Program Against Alcohol Consumption in Early Adolescence. Journal of Early Adolescence, 32(2), 200-225. https://doi. org/10.1177/0272431610384489

Esta obra está bajo: Creative commons attribution 4.0 international license. El beneficiario de la licencia tiene el derecho de copiar, distribuir, exhibir y representar la obra y hacer obras derivadas siempre y cuando reconozca y cite la obra de la forma especificada por el autor o el licenciante.

\section{(cc) BY}

